\title{
Prediction of HER2 gene status in Her2 $2+$ invasive breast cancer: a study of 108 cases comparing ASCO/CAP and FDA recommendations
}

\author{
Frédéric Chibon ${ }^{1}$, Isabelle de Mascarel $^{1}$, Ghislaine Sierankowski ${ }^{1}$, Véronique Brouste ${ }^{2}$, \\ Hervé Bonnefoi ${ }^{3}$, Marc Debled ${ }^{3}$, Louis Mauriac ${ }^{3}$ and Gaëtan MacGrogan ${ }^{1}$ \\ ${ }^{1}$ Department of Pathology, Institut Bergonié, Bordeaux, France; ${ }^{2}$ Department of Biostatistics, Institut \\ Bergonié, Bordeaux, France and ${ }^{3}$ Department of Medical Oncology, Institut Bergonié, Bordeaux, France
}

\begin{abstract}
Most Her2 testing guidelines recommend that all cases scoring Her2 $2+$ by immunohistochemistry should be analyzed by fluorescent in situ hybridization (FISH) to determine HER2 status to confirm eligibility for Trastuzumab therapy in breast cancer. The aim of our study was to determine HER2 gene and chromosome 17 (CEN17) status in a series of 108 Her2 2 + consecutive cases and study the correlation between pathological characteristics of the tumors and HER2 amplification. Invasive breast cancers were tested by FISH using the Dako HER2 FISH pharmDx ${ }^{\circledR}$ kit. The Her2 immunohistochemistry protocol was performed using the polyclonal A0485 antibody (Dako ${ }^{\circledR}$ ) diluted to 1:1500. HER2 and CEN17 status were correlated to tumor SBR grade, mitotic count, estrogen receptor, progesterone receptor status and percentage of Her2 immunohistochemistry-positive cells. Following Food and Drug Administration guidelines, ie, HER2/CEN17 ratio $\geq 2$ and an HER2 copy number $>4$, amplified cases were observed in $36(33 \%)$ and 49 (45\%) cases, respectively, and following American Society of Clinical Oncology/College of American Pathologists guidelines, ie, HER2/CEN17 ratio > 2.2 and an HER2 copy number $>6$, amplified cases represented 30 and $24 \%$ of the study population, respectively. Chromosome 17 polysomy (CEN17 $>2.25)$ was observed in $39(36 \%)$ tumors. Significant positive correlations were found between FISH HER2 amplified cases and Her2 immunostaining $>60 \%\left(P=1.1 .10^{-5}\right)$, SBR grade 3 $(P=0.0001)$, nuclear atypia $(P=0.03)$ and mitotic count $(P=0.008)$. By multivariate analysis, Her2 immunostaining $>60 \%\left(P<10^{-3}\right)$ and SBR grade $3\left(P<10^{-3}\right)$ were independent factors predicting $H E R 2$ amplification status irrespective to cutoff guidelines. All SBR grade 3 cases with more than $60 \%$ Her2 + cells had an HER2/CEN17 ratio $\geq 2$, only one had a ratio $\leq \mathbf{2 . 2}$. In our series of consecutive Her2 $2+$ cases, one-third demonstrated $H E R 2$ amplification, and one-third had chromosome 17 polysomy. Pathological factors, in particular SBR grade 3 and more than $60 \%$ Her 2 cells, were significantly correlated with HER2 amplification.
\end{abstract}

Modern Pathology (2009) 22, 403-409; doi:10.1038/modpathol.2008.195; published online 5 December 2008

Keywords: HER2; FISH; immunohistochemistry; amplification

The HER2 gene encodes an epidermal growth factor receptor tyrosine kinase that is overexpressed in $10-30 \%$ of invasive breast cancers. Her2 overexpression occurs primarily through amplification of the wild-type HER2 gene and is associated with poor disease-free survival and resistance to certain chemotherapeutic agents. ${ }^{1}$ Her2 overexpression and amplification are predictive of response to therapy with Trastuzumab (Herceptin ${ }^{\circledR}$; Hofman La Roche, Bâle), ${ }^{2}$ determination of Her2 status in breast cancer

Correspondence: F Chibon, PhD, Department of Pathology, Institut Bergonié, 229 cours de l'Argonne, Bordeaux cedex 33076, France. E-mail: chibon@bergonie.org

Received 9 September 2008; revised 23 October 2008; accepted 3 November 2008; published online 5 December 2008 is therefore important. The two most widespread used methods for determining Her2 status, in routine practice, include immunohistochemistry and fluorescent in situ hybridization (FISH). Immunohistochemistry is used as a screening method to determine the level of Her2 protein expression in breast cancers and Her2 immunohistochemical results are generally expressed in a four scale scoring system ranging from 0 to $3+.{ }^{3}$ Scoring criteria in this system comprise percentage of positive tumor cells and quality of staining including intensity of staining and type of membrane staining. According to clinical trials, expert consensus and US Food and Drug Administration (FDA) and the recent American Society of Clinical Oncology/College of American Pathologists (ASCO/CAPs) 
recommendations, ${ }^{4}$ an invasive breast cancer with an Her2 $3+$ score is considered a positive Her2 tumor, ie, the patient is eligible for Trastuzumab therapy. Moreover, samples scored as Her2 $2+$ are considered Her2 equivocal and should be further tested with a validated assay for HER2 gene amplification, such as FISH, before considering for Trastuzumab therapy. FISH is considered the gold standard for HER2 status determination in breast cancer. Determination of HER2 status by FISH can either be done by assessing the mean number of HER2 copies or by calculating the mean HER2/ CEN17 ratio in a population of tumor nuclei, where CEN17 represents the number of chromosomes 17. Comparison of Her2 status determined by immunohistochemistry and FISH has been previously performed in numerous studies. Using a cutoff of $60 \%$ of positive tumor cells, we had previously found a $95 \%$ concordance rate between Her2 immunohistochemistry and FISH in a series of 119 cases of invasive breast carcinomas. ${ }^{5}$ However, data on HER2 FISH or chromosome 17 polysomy statuses are sparse for breast tumors showing equivocal Her2 immunostaining. The ASCO/CAP have recently introduced more stringent criteria defining HER2 amplification $^{3}$ as compared to the FDA criteria formerly used and data on the consequences of such a change are sparse. ${ }^{6}$

HER2 amplification as well as chromosome 17 polysomic statuses are known to be associated with adverse pathological and clinical factors in breast cancer $^{7}$ and therefore assessment of such factors in the presence of an equivocal Her2 immunostaining, may help predict HER2 amplification or chromosome 17 polysomy.

The aim of the present study was therefore to assess the HER2 gene and chromosome 17 status in Her2 $2+$ invasive breast cancers, according to the FDA and the ASCO/CAP criteria and to determine if tumor pathological factors and percentage of Her2 $2+$ cells could predict these different conditions.

\section{Materials and methods}

Between 08 January 2005 and 08 January 2006, 275 cases of invasive breast cancer were assessed for their HER2 gene status by the FISH assay at the Pathology Department of Institut Bergonié. These cases were either sent to the Department by external laboratories or were in-house cases. The FISH assay for determination of HER2 status was mainly performed for cases with equivocal Her2 $2+$ immunostaining. However cases with no or high Her2 overexpression were also tested by FISH analysis, for control or confirmation of Her2 status, requested by the patient's physician.

For the purpose of this study, Her2 immunohistochemistry was systematically reperformed on all cases tested by FISH and Her2 expression using the Herceptest score was reassessed by one of the authors (GMG). Of 275 cases, 108 presented with a definite Her $2+$ score and selected for this study. For each case, the original H\&E slides and estrogen and progesterone receptor immunostains were requested and the histological type, Elston and Ellis tumor grade, mitotic count, estrogen and progesterone receptor status were assessed.

\section{Her2 Immunohistochemistry}

For all the cases tested for Her2 overexpression, antigen retrieval was performed by heating $5 \mu \mathrm{m}$ tissue sections in $0.01 \mathrm{M}$ citrate buffer $\mathrm{pH}=6$ in a pressure cooker for $20 \mathrm{~min}$. Tissue sections were then incubated for $60 \mathrm{~min}$ with a polyclonal antiHer2 antibody (Dako ${ }^{\circledR}$, Trappes France, AO485 dilution 1:1500). A labeled streptavidin-biotinperoxydase method (LSAB kit K5001, Dako ${ }^{\mathbb{R}}$, France) was used for visualization of the immunoreactions with Diaminobenzidine as a chromogen.

\section{Her2 Immunohistochemical Scoring}

The Herceptest scoring system was used to assess Her2 overexpression. Briefly, cases showing no membrane immunostaining or in less than $10 \%$ invasive cancer cells were scored $0+$, cases with weak and incomplete membrane staining in more than $10 \%$ of invasive cancer cells were scored $1+$, cases with complete membrane staining that was either nonuniform or weak in intensity but with obvious circumferential distribution in at least $10 \%$ of cells were scored $2+$ and finally cases with strong membrane staining in more than $10 \%$ invasive tumor cells were scored $3+$ (Dako A/S G, Denmark:HercepTest package insert: http://pri.dako. com/28630_herceptest_interpretation_manual.pdf). In each case, negative benign glands were used as internal controls for specificity of the immunoreaction and an invasive breast cancer case with known Her2 overexpression was used as an external control for sensitivity of the immunoreaction. For the purpose of this study, the percentage of positive Her2 invasive cancer cells and intensity of staining were also assessed.

\section{HER2 FISH}

Assessment of the HER2 gene and chromosome 17 status was performed using the DAKO HER2 PharmDX ${ }^{\mathbb{R}}$ kit following the manufacturer's instructions. After deparaffinization and rehydration, specimens were heated in the pretreatment solution provided in the kit for 10 min. Proteolytic digestion was carried out with ready-to-use Pepsin at room temperature for 5-15 min. A ready-to-use probe mix consisting of a mixture of Texas red-labeled DNA probes covering a $218 \mathrm{~kb}$ region including HER2 gene on chromosome 17, and a mixture of 
fluorescein-labeled PNA probes targeted at the centromeric region of chromosome 17 (CEN17) was then applied. After $5 \mathrm{~min}$ denaturation at $82^{\circ} \mathrm{C}$, slides and probe mix were incubated overnight at $45^{\circ} \mathrm{C}$ in a humidified hybridization chamber. The following morning, after a series of stringent washes, a fluorescence mounting medium containing DAPI was then applied.

\section{HER2 FISH Interpretation}

The FISH specimens were analyzed on a Nikon Eclipse 80i fluorescent microscope with appropriate filters and pictures were captured using a Hamamatsu C4742-95 CCD camera and analyzed with the Genikon ${ }^{\circledR}$ software (Alphelys ${ }^{\mathbb{R}}$, France). In each case, a minimum number of 40 invasive tumor cell nuclei were analyzed in at least two distinct areas. The mean number of HER2 copies and the mean number of CEN17 per nucleus were then calculated and the HER2/CEN17 ratio was established. In this study, a case was considered HER2 amplified if the HER2/CEN17 was equal to or more than two following FDA or more than 2.2 following ASCO/ CAP guidelines, ${ }^{3}$ respectively. In borderline cases, ie, showing $1.8<H E R 2 / C E N 17<2.2$, an additional number of 20 invasive tumor cell nuclei were analyzed and the mean number of HER2 copies and CEN17 and the HER2/CEN17 ratio were established in a total of 60 nuclei.

\section{Statistical Analysis}

$\chi^{2}$-Tests were performed to evaluate the relationship between different tumor pathologic features, including estrogen receptor and progesterone receptor status, tumor grade, percentage of Her2 $2+$ invasive tumor cells and HER2 amplification status or chromosome 17 polysomic status. Reciprocal influence among the different predictive factors was determined by multivariate analysis using a backward logistic regression test. The variables that predicted chromosome 17 polysomy in the first analysis and HER2 amplification in the second analysis were determined. All factors were included in the logistic regression analyses, irrespective of their $P$-value by univariate analysis; but only those with a $P$-value $\leq 5 \%$ were retained in the final models. Univariate and multivariate analyses (logistic regression model) were performed using SAS software version 8 .

\section{Results}

HER2 Amplification and Chromosome 17 Polysomy in the Series According to FDA and ASCO/CAP Guidelines

Distribution of mean HER2 copy number per nucleus and mean HER2/CEN17 ratio per nucleus in the study population of 108 Her $2+$ cases are
Table 1 Distribution of mean HER2 copy number per nucleus and mean HER2/CEN17 ratio per nucleus in a series of 108 Her2 $2+$ cases of invasive breast cancer

\begin{tabular}{|c|c|c|c|c|}
\hline & & \multicolumn{2}{|c|}{ HER2 } & \multirow[t]{2}{*}{ Total } \\
\hline & & $\leq 4$ & $>4$ & \\
\hline \multicolumn{5}{|c|}{ (A) Following the FDA recommendations } \\
\hline \multirow[t]{2}{*}{ HER2/CEN17 } & $<2$ & $59(55 \%)$ & $13(12 \%)$ & $72(67 \%)$ \\
\hline & $\geq 2$ & 0 & $36(33 \%)$ & $36(33 \%)$ \\
\hline Total & & $\begin{array}{c}59(55 \%) \\
\leq 6\end{array}$ & $\begin{array}{c}49(45 \%) \\
>6\end{array}$ & $108(100 \%)$ \\
\hline \multicolumn{5}{|c|}{ (B) Following the ASCO/CAP recommendations } \\
\hline \multirow[t]{2}{*}{ HER2/CEN17 } & $\leq 2.2$ & $73(68 \%)$ & $3(3 \%)$ & $76(71 \%)$ \\
\hline & $>2.2$ & $9(8 \%)$ & $23(21 \%)$ & $32(29 \%)$ \\
\hline Total & & $82(76 \%)$ & $26(24 \%)$ & $108(100 \%)$ \\
\hline
\end{tabular}

FDA, Food and Drug Administration; ASCO/CAP, American Society of Clinical Oncology/College of American Pathologist.

shown in Table 1. Depending on the FDA and ASCO/CAP guidelines, $36(33 \%)$ and $32(30 \%)$, respectively, of the Her2 $2+$ cases were considered HER2 amplified in the series. All cases with a HER2/ CEN17 ratio equal to or more than two had more than four HER2 copies per nucleus. Moreover, 13 cases $(12 \%)$ with more than four HER2 copies per nucleus showed a HER2/CEN17 ratio less than two. Results were slightly different considering the ASCO/CAP cutoffs, because nine cases with a ratio more than 2.2 had less than six HER2 copies per nucleus and only three cases with more than six HER2 copies per nucleus showed a HER2/CEN17 ratio equal to or less than 2.2 .

Using 2.25 CEN17 per nucleus as the cutoff for defining polysomy in the series, 39 (36\%) Her2 $2+$ cases showed chromosome 17 polysomy.

All the 13 cases with more than four HER2 copies but with a HER2/CEN17 ratio of less than two per nucleus demonstrated CEN17 polysomy, with mean CEN17 varying between 2.3 and 4 . In the same cases, HER2 copy numbers varied from 4.16 to 6.71 with two cases presenting with more than six HER2 copies per nucleus. Corresponding HER2/CEN17 ratios varied from 1.23 to 1.92 , with five cases presenting with an equivocal HER2 status with a ratio of more than 1.8 .

\section{Association Between Chromosome 17 Polysomic Status and Tumor Pathologic Features}

Using the $\chi^{2}$-test, in this series of 108 Her $2+$ cases, a significant negative association between chromosome 17 polysomy and more than $60 \%$ Her $22+$ invasive tumor cells was found $(P=0.05)$. Chromosome 17 polysomy tended to be more often present in tumors with less than $60 \%$ invasive tumor cells showing Her2 $2+$ intensity of staining.

No significant correlation was found between chromosome 17 polysomic status and other pathologic variables such as tumor grade, mitotic count, 
Table 2 Associations between pathologic criteria and HER2 gene status, using the $\chi^{2}$-test

\begin{tabular}{lcc}
\hline Pathologic criteria & \multicolumn{2}{c}{ HER2 /CENT17 } \\
\cline { 2 - 3 } & $\begin{array}{c}F D A \\
\text { ratio } \geq 2\end{array}$ & $\begin{array}{c}\text { ASCO 2007 } \\
\text { ratio }>2.2\end{array}$ \\
\hline Her2 + cells $>60 \%$ & $1.10^{-5}$ & $1.10^{-5}$ \\
Grade & $1.10^{-5}$ & $1.10^{-3}$ \\
Mitotic count & 0.008 & 0.03 \\
Nuclear atypia & 0.03 & NS \\
Differentiation & NS & NS \\
IHC estrogen receptor & NS & NS \\
IHC progesterone receptor & NS & NS \\
\hline
\end{tabular}

NS, not significant; FDA, Food and Drug Administration; ASCO, American Society of Clinical Oncology; IHC, immunohistochemistry.

tumor differentiation, nuclear atypia, estrogen receptor and progesterone receptor status.

\section{Association Between HER2 Amplification Status and Tumor Pathologic Features}

Using the HER2/CEN17 $\geq 2$ or the HER2/CEN17 $>2.2$ ratios for defining HER2 amplified tumors, a strong positive correlation was found between the percentage of Her2 $2+$ invasive tumor cells and HER2 gene status. Tumors with more than $60 \% 2+$ invasive tumor cells were more often HER2 amplified than the others $\left(P=1.10^{-5}\right)$ with both cutoffs. Furthermore, a significant positive correlation, again with both cutoffs, was found between tumor grade, mitotic count and HER2 amplification (Table 2) and a positive correlation was found with nuclear atypia only following FDA guidelines. Finally, no significant association was found between hormonal receptor status and HER2 status whatever the cutoff value used. By multivariate analysis, using the HER2/CEN17 FDA cutoff at two, two factors independently predicted HER2 status, ie, percentage of Her2 $2+$ invasive tumor cells $\geq 60 \%$ (odd ratio $=12 ; 95 \%$ CI: $3.2-44.5 ; P<10^{-3}$ ) and tumor grade 3 (odd ratio $=6$; 95\% CI: $2.3-16.7 ; P<10^{-3}$ ). All grade 3 invasive breast cancers presenting with more than $60 \%$ Her2 $2+$ invasive tumor cells $(n=8)$ showed a HER2/CEN17 ratio of more than two. Using the HER2/CEN17 ASCO/CAP cutoff at 2.2, again the same two factors independently predicted HER2 status, ie, percentage of Her2 $2+$ invasive tumor cells $\geq 60 \%$ (odd ratio $=9.8$; $95 \%$ CI $2.9-33.6$; $P<10^{-3}$ ) and tumor grade 3 (odd ratio $=4.5 ; 95 \%$ CI $1.7-12 ; P=0.003$ ) and all but one case of grade 3 invasive breast cancers presenting with more than $60 \%$ Her2 $2+$ invasive tumor cells had a HER2/ CEN17 ratio of more than 2.2 (Table 3).

\section{Discussion}

HER2 status is relevant for patient care and overexpression of HER2 indicates a poor outcome. ${ }^{8-10}$
The proper algorithm for the assessment of HER2 status has yet to be defined but evidence arising from comparative studies shows that gene amplification correlates better with prognosis and may be more accurate to identify patients who are likely to benefit from Herceptin ${ }^{\circledR}$ treatment. ${ }^{11-13}$ Owing to different drawbacks associated with FISH (cost, time consuming, not routinely performed in all laboratories), immunohistochemistry represents the first screening step and FISH should be performed in all cases where immunostaining is doubtful (technical artifact) or equivocal (read as $2+$ ). FISH being also subject to artifacts, it is therefore of interest to determine histological criteria allowing prediction of the HER2 gene status established by FISH.

In our study, we analyzed 108 Her2 $2+$ tumors by FISH with the aim to identify which pathologic tumor factor might predict HER2 gene status in tumors showing equivocal Her2 staining. The ASCO/CAP having moved to more stringent cutoff values than the FDA, we analyzed our data taking into account the different criteria defining a HER2 amplified tumor. Considering positive cases when HER2/CEN17 ratio is $\geq 2$, the two-thirds (67\%) of the samples in our series would have been declared as negative and therefore not eligible for Herceptin ${ }^{\mathbb{R}}$ treatment. This rate is very close to the one that would have been obtained using the HER2/CEN17 ratio $>2.2(70 \%)$. According to the literature, this rate is between 64 and $93 \%$ depending on the samples analyzed and on the decision criteria used, ratio or HER2 copy number. ${ }^{9,14}$ Even if very few studies have been carried out considering only Her2 $2+$ cases, Merola et $a l^{7}$ report a negative rate of $70 \%$ in such a group. Generally, the false-positive rate of the immunohistochemistry appears to be around $70 \%$. This very high rate can be explained by genetic mechanisms or technical pitfalls.

First, in our series and others, ${ }^{15,16}$ the HER2 copy number in the majority of Her2 $2+$ amplified cases is lower than 10 unclustered copies (mean $=6.95$ ) instead of more than 10 copies in cases scored $3+$ (data not shown and 15) and in negative cases this copy number is 2.9 as a mean. According to cytogenetic criteria, there is a great difference between gain and amplification. The last is defined as an increase in copy number of a restricted region of a chromosome arm generated by breakage-fusionbridge cycle. Amplified DNA resides either on extrachromosomal circles of DNA that lack centromeres, called double minutes, or as a cytologically visible chromosomal expansion, ${ }^{17,18}$ both presenting in interphase FISH as clustered signals of more than 10 copies (Figure 1a). In our study, most of the Her 2 $2+$ cases with a ratio $>2.2$ harbored spread FISH signals and should so be considered as gain instead of true amplification of the HER2 locus. Even if this gain (from 4 to 10 copies) could explain the weak expression detected in cases scored $2+$ in immunohistochemistry and not amplified or borderline, growing evidence is demonstrating that all these 
Table 3 Distribution of mean HER2/CEN17 ratio per nucleus according to tumor grade and percentage of Her2 2+ cells

\begin{tabular}{|c|c|c|c|}
\hline \multirow[t]{2}{*}{ Tumor grade and percentage of IHC Her $2+$ cells } & \multicolumn{2}{|c|}{ HER2/CEN17 } & \multirow[t]{2}{*}{ Total } \\
\hline & $<2$ & $\geq 2$ & \\
\hline \multicolumn{4}{|l|}{ (A) Following the FDA recommendations } \\
\hline Nongrade 3 and $<60 \%$ & $51(48 \%)$ & $9(9 \%)$ & $60(57 \%)$ \\
\hline Nongrade 3 and $\geq 60 \%$ & $4(4 \%)$ & $6(5 \%)$ & $10(9 \%)$ \\
\hline Grade 3 and <60\% & $15(14 \%)$ & $13(12 \%)$ & $28(26 \%)$ \\
\hline Grade 3 and $\geq 60 \%$ & 0 & $8(8 \%)$ & $8(8 \%)$ \\
\hline Total & $70(66 \%)$ & $36(34 \%)$ & $106(100 \%)$ \\
\hline & $\leq 2.2$ & $>2.2$ & \\
\hline \multicolumn{4}{|l|}{ (B) Following the ASCO/CAP recommendations } \\
\hline Nongrade 3 and $<60 \%$ & $52(49 \%)$ & $8(7 \%)$ & $60(56 \%)$ \\
\hline Nongrade 3 and $\geq 60 \%$ & $4(4 \%)$ & $6(6 \%)$ & $10(10 \%)$ \\
\hline Grade 3 and $<60 \%$ & $17(16 \%)$ & $11(10 \%)$ & $28(26 \%)$ \\
\hline Grade 3 and $\geq 60 \%$ & $1(1 \%)$ & $7(7 \%)$ & $8(8 \%)$ \\
\hline Total & $74(70 \%)$ & $32(30 \%)$ & $106(100 \%)$ \\
\hline
\end{tabular}

IHC, immunohistochemistry; FDA, Food and Drug Administration; ASCO/CAP, American Society of Clinical Oncology/College of American Pathologist.
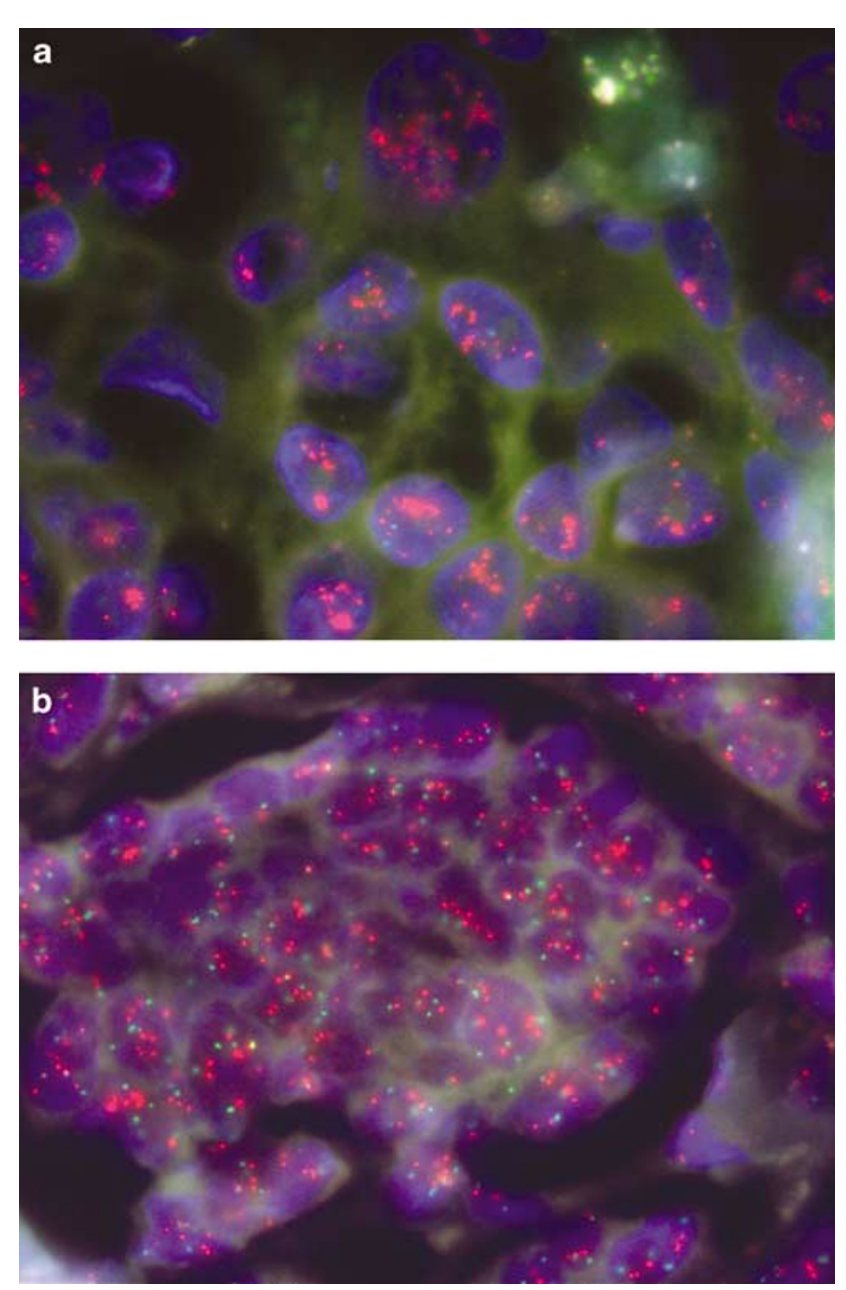

Figure 1 HER2/CEN17 FISH on two Her2 $2+$ invasive breast cancer. Red signal corresponds to HER2 probe and green signal to CEN17 probe. (a) Amplified case harboring more than 20 clustered HER2 copies per nucleus. (b) Polysomic case presenting with a mean 3.38 CEN17 copies and 6.71 HER2 copies per nucleus. low-grade cases should probably be considered as negative for HER2 amplification. ${ }^{8}$ Arnould et al ${ }^{13}$ showed that tumors with a low HER2 amplification (less than 10 copies per nucleus) had a significant lower rate of complete pathological response to Trastuzumab $^{\circledR}$ neoadjuvant therapy than those with high amplification (more than 10 copies per nucleus). In line with this, the new ASCO/CAP guidelines now consider that at least six HER2 copies per nucleus are needed to declare a case as positive, and may be a step forward would be to take into account also the HER2 signal organization, cluster or spread, to declare a case as truly 'amplified' as compared to a case 'eligible' for Herceptin ${ }^{\circledR}$ treatment.

Second, it appears also from few published studies,${ }^{14}$ detecting an abnormal low rate of amplified cases in the Her $2+$ population $(7.14 \%)$ that the immunohistochemical conditions may be too sensitive and not specific enough. It could so be of interest to establish standard immunohistochemical conditions and interpretation to render it more specific of HER2 real amplification, meaning Her2 $2+$ with at least six or eight copies.

Finally, this weak expression, negative for amplification in $70 \%$ of the cases, could also be explained, at least in part, by chromosome 17 polysomy. ${ }^{19}$ In our series, $40 \%$ of the nonamplified cases were polysomic for chromosome 17 (Figure 1b). This mechanism, ie, polysomy, is truly different from amplification in which gene expression can be modulated not only because of the copy number but also by the new genomic environment related to amplification. ${ }^{20-22}$ Nevertheless, using 2.25 CEN17 per nucleus as the cutoff for defining polysomy, $36 \%$ of Her2 $2+$ cases showed chromosome 17 polysomy. The $\chi^{2}$-test demonstrated that polysomy 17 is statistically more frequently observed in cases with less than $60 \%$ of Her2 $2+$ tumor cells and 
without gene amplification. Impact of polysomy 17 on HER2 expression and response to Herceptin ${ }^{\circledR}$ is still controversial, but it appears from our results and others that the mechanism for HER2 amplification is independent of polysomy. ${ }^{14,23}$ Following this hypothesis, polysomy could be a surrogate of a general chromosomal instability that is well known to be predictive of poor clinical outcome. ${ }^{24}$ In line with this, Salido et $a l^{23}$ found that nodal involvement was more frequent in polysomic than in nonpolysomic cases.

However, in the polysomic group of our series, 13 invasive breast cancers had more than four HER2 copies per nucleus, but 10 of these cases had less than six HER2 copies per nucleus (Table 1) and might be declared as negative for amplification following ASCO/CAP guidelines. The remaining three cases should so be considered with regard to the other pathological and clinical data to determine the eligibility to Herceptin ${ }^{\circledR}$ treatment.

Predictive histologic criteria depend on the HER2/CEN17 cutoff value used. Considering the less stringent cutoff (ie; ratio $\geq 2$ ), all the positive cases, in our series, had more than four HER2 copies per nucleus. Two major pathologic features, tumor grade and the percentage of Her2 $2+$ invasive tumor cells were independently predictive of HER2 status and, following our results, an Her2 $2+$ grade 3 invasive breast cancer had $58 \%$ of probability to be eligible for Herceptin ${ }^{\circledR}$ treatment, a tumor with more than $60 \%$ Her2 $2+$ invasive tumor cells had a probability of $77 \%$ to be eligible and a tumor harboring both criteria had $100 \%$ of probability to be positive for HER2 amplification. With the more stringent cutoff (ie, ratio $>2.2$, not only the pathologic factors still demonstrated an independent predictive value but also became more efficient to predict a nonamplified case. Indeed, cases with less than $60 \% 2+$ cells and histologic grade 1 or 2 harbored less than six HER2 copies per nucleus or a ratio $<2.2$ in 93.3 and $86.7 \%$ of the cases, respectively.

Taken together, our data suggest that a proper algorithm for Herceptin ${ }^{\circledR}$ therapy decision in cases scored Her2 $2+$ should take into account not only FISH (with respect to ASCO/CAP guidelines) or immunohistochemistry but a combination of tumor grading (grades 1-2 vs grade 3), immunohistochemistry (less or more than $60 \%$ Her2 $2+$ invasive tumor cells) and FISH (less or more than six copies, clustered or not).

\section{Acknowledgements}

This work was supported by grants from the Comités départementaux de la Ligue Nationale de Lutte contre le Cancer de la Gironde, la Charente, la Charente-Maritime, la Dordogne et des Landes and from the 'Lyon's club de Bergerac'.

\section{Disclosure/conflict of interest}

The authors declare no conflict of interest.

\section{References}

1 Nahta R, Esteva FJ. Herceptin: mechanisms of action and resistance. Cancer Lett 2006;232:123-138.

2 Hofmann M, Stoss O, Gaiser T, et al. Central HER2 IHC and FISH analysis in a trastuzumab (Herceptin) phase II monotherapy study: assessment of test sensitivity and impact of chromosome 17 polysomy. J Clin Pathol 2008;61:89-94.

3 Wolff AC, Hammond ME, Schwartz JN, et al. American Society of Clinical Oncology/College of American Pathologists guideline recommendations for human epidermal growth factor receptor 2 testing in breast cancer. J Clin Oncol 2007;25:118-145.

4 Slamon DJ, Leyland-Jones B, Shak S, et al. Use of chemotherapy plus a monoclonal antibody against HER2 for metastatic breast cancer that overexpresses HER2. N Engl J Med 2001;344:783-792.

5 Vincent-Salomon A, MacGrogan G, Couturier J, et al. Calibration of immunohistochemistry for assessment of HER2 in breast cancer: results of the French multicentre GEFPICS study. Histopathology 2003;42: 337-347.

6 Brunelli M, Manfrin E, Martignoni G, et al. HER-2/neu assessment in breast cancer using the original FDA and new ASCO/CAP guideline recommendations: impact on selecting patients for herceptin therapy. Am J Clin Pathol 2008;129:907-911.

7 Merola R, Mottolese M, Orlandi G, et al. Analysis of aneusomy level and HER-2 gene copy number and their effect on amplification rate in breast cancer specimens read as $2+$ in immunohistochemical analysis. Eur J Cancer 2006;42:1501-1506.

8 Ross JS, Fletcher JA, Bloom KJ, et al. Targeted therapy in breast cancer: the HER-2/neu gene and protein. Mol Cell Proteomics 2004;3:379-398.

9 Winston JS, Ramanaryanan J, Levine E. HER-2/neu evaluation in breast cancer are we there yet? Am J Clin Pathol 2004;121(Suppl):S33-S49.

10 Goldhirsch A, Gelber RD. Life with consequences of breast cancer: pregnancy during and after endocrine therapies. Breast 2004;13:443-445.

11 Mass RD, Press MF, Anderson S, et al. Evaluation of clinical outcomes according to HER2 detection by fluorescence in situ hybridization in women with metastatic breast cancer treated with trastuzumab. Clin Breast Cancer 2005;6:240-246.

12 Tubbs RR, Pettay JD, Roche PC, et al. Discrepancies in clinical laboratory testing of eligibility for trastuzumab therapy: apparent immunohistochemical false-positives do not get the message. J Clin Oncol 2001;19: 2714-2721.

13 Arnould L, Arveux P, Couturier J, et al. Pathologic complete response to trastuzumab-based neoadjuvant therapy is related to the level of HER-2 amplification. Clin Cancer Res 2007;13:6404-6409.

14 Torrisi R, Rotmensz N, Bagnardi V, et al. HER2 status in early breast cancer: relevance of cell staining patterns, gene amplification and polysomy 17. Eur J Cancer 2007;43:2339-2344.

15 Lal P, Salazar PA, Ladanyi M, et al. Impact of polysomy 17 on HER-2/neu immunohistochemistry in breast 
carcinomas without HER-2/neu gene amplification. J Mol Diagn 2003;5:155-159.

16 Lal P, Salazar PA, Hudis CA, et al. HER-2 testing in breast cancer using immunohistochemical analysis and fluorescence in situ hybridization: a singleinstitution experience of 2279 cases and comparison of dual-color and single-color scoring. Am J Clin Pathol 2004;121:631-636.

17 McClintock B. The stability of broken ends of chromosomes in Zea Mays. Genetics 1941;26:234-282.

18 Debatisse M, Coquelle A, Toledo F, et al. Gene amplification mechanisms: the role of fragile sites. Recent Results Cancer Res 1998;154:216-226.

19 Ma Y, Lespagnard L, Durbecq V, et al. Polysomy 17 in HER-2/neu status elaboration in breast cancer: effect on daily practice. Clin Cancer Res 2005;11:4393-4399.

20 Bartlett JM, Going JJ, Mallon EA, et al. Evaluating HER2 amplification and overexpression in breast cancer. J Pathol 2001;195:422-428.
21 Downs-Kelly E, Yoder BJ, Stoler M, et al. The influence of polysomy 17 on HER2 gene and protein expression in adenocarcinoma of the breast: a fluorescent in situ hybridization, immunohistochemical, and isotopic mRNA in situ hybridization study. Am J Surg Pathol 2005;29:1221-1227.

22 Dal LL, Durbecq V, Desmedt C, et al. Correction for chromosome-17 is critical for the determination of true Her-2/neu gene amplification status in breast cancer. Mol Cancer Ther 2006;5:2572-2579.

23 Salido M, Tusquets I, Corominas JM, et al. Polysomy of chromosome 17 in breast cancer tumors showing an overexpression of ERBB2: a study of 175 cases using fluorescence in situ hybridization and immunohistochemistry. Breast Cancer Res 2005;7:R267-R273.

24 Carter SL, Eklund AC, Kohane IS, et al. A signature of chromosomal instability inferred from gene expression profiles predicts clinical outcome in multiple human cancers. Nat Genet 2006;38:1043-1048. 